\title{
Módulo educativo Socializarte en las habilidades sociales avanzadas de estudiantes de Ciencias de la Comunicación
}

\section{Educational module Socializarte in advanced social skills of students of Communication Sciences}

\author{
Rubén Darío Alania Contreras ${ }^{1, *}$ \\ ${ }^{1}$ Escuela Académico Profesional de Ciencias y Tecnologías de la Comunicación \\ Facultad de Humanidades, Universidad Continental
}

\section{RESUMEN}

El estudio responde a la necesidad de reforzar las habilidades sociales avanzadas en estudiantes de Ciencias de la Comunicación como factor importante para su desarrollo profesional. La investigación de diseño cuasi experimental tuvo el objetivo del demostrar la influencia de la aplicación del módulo educativo Socializarte en las habilidades sociales avanzadas de estudiantes del segundo y tercer semestre de la Facultad de Ciencias de la Comunicación de la Universidad Nacional del Centro del Perú de Huancayo en 2016. Se tuvo como muestra a 30 estudiantes en el grupo experimental y 30 estudiantes en el grupo control; en el grupo experimental se aplicó el módulo educativo Socializarte, diseñado para la investigación; como instrumento de recolección de datos en el pre test y post test se utilizó el cuestionario de habilidades sociales avanzadas, diseñado para la investigación. En los resultados se comprobó que en el pre test, no existen diferencias significativas entre las medias de las habilidades sociales avanzadas de los grupos experimental y control ( $T=0,29$ y $p=0,773)$; se evidenció que, en el post test, existen diferencias significativas entre las medias de las habilidades sociales avanzadas de los grupos experimental y control, favorables al grupo experimental ( $T=13,93$ у $\mathrm{p}=0$ ). La investigación concluyó que la influencia del módulo educativo Socializarte en las habilidades sociales avanzadas de los estudiantes del segundo y tercer semestre de la Facultad de Ciencias de la Comunicación, fue significativa.

Palabras clave: Habilidades sociales, módulo educativo, psicopedagogía.

\begin{abstract}
The study responds to the need to reinforce social skills advanced students in communication sciences as an important factor for their profesional development. Quasi-experimental design research had the objective of showing the influence of the application of the educational module socialize in advanced social skills of students of the second and third semester of the Faculty of Communication Sciences of the Universidad Nacional del Centro del Peru from Huancayo in 2016. It was a sample to 30 students in the experimental group and 30 students in the control group; in the experimental group applied the educational module socialize, designed for research; advanced social skills questionnaire, designed for research was used as instrument of collection of data in the pre test and post test. The results showed that in the pre test, there are no significant differences between the averages of the advanced social skills in the experimental and control groups $(T=0,29$ and $p=0,773)$; it was evident that, in the post test, there are significant differences between the average of the experimental and control groups advanced social skills,favorable to the experimental group $(T=13,93$ and $p=0)$. The investigation concluded that you influence educational module socialize in advanced social skills of students in the second and third semester of the faculty of communication sciences, was significant.
\end{abstract}

Keywords: Social skills, educational module, educational psychology.

Historial del artículo:

Recibido, 04 de marzo 2017; aceptado, 10 de mayo de 2017; disponible en línea, 05 de enero de 2018

* Doctor en Educación, docente la Escuela Académico Profesional de Ciencias y Tecnologías de la Comunicación de la Universidad Continental de Huancayo.

Correo: ralania@continental.edu.pe 


\section{INTRODUCCIÓN}

Los enfoques educativos cada vez dan más valor al desarrollo de competencias sociales (Segura, 2007), educadores y psicólogos están de acuerdo en que, al momento de relacionarse, una persona requiere pensar, reconocer y controlar emociones y manifestar valores morales básicos. Educar a un ser humano no consiste en saturarlo solo de conocimiento; lo imprescindible y absolutamente básico es convertirlo en persona. En ese sentido el enfoque educativo debe ser cognitivo, emocional y moral.

El presente estudio responde a la necesidad de reforzar las habilidades sociales avanzadas en estudiantes de Ciencias de la Comunicación de la Universidad Nacional del Centro del Perú de Huancayo. Las habilidades sociales avanzadas son capacidades que en profesionales de ciencias de la comunicación deberían ser sobresalientes, el perfil del ingresante, estudiante y egresado de la carrera de Ciencias de la Comunicación de la Universidad Nacional del Centro del Perú requiere un alto desempeño de habilidades sociales avanzadas; sin embrago, en la práctica existen muchas debilidades.

Los antecedentes históricos de la facultad refieren varios periodos de conflicto que involucraron docentes y estudiantes; los grupos de interés reclaman adecuadas competencias sociales de los egresados y los informes del área tutoría de la Facultad de Ciencias de la Comunicación, junto a investigaciones exploratorias previas, manifiestan una seria debilidad en la formación integral de sus educandos. Como propuesta a tal situación, en la presente investigación se diseñó y aplicó un módulo educativo de entrenamiento en habilidades sociales avanzadas al que se denominó Socializarte.

Así, se planteó como problema principal la interrogante: ¿Cómo influye la aplicación del módulo Socializarte en las habilidades sociales avanzadas de estudiantes del segundo y tercer semestre de la Facultad de Ciencias de la Comunicación de la Universidad Nacional del Centro del Perú de Huancayo - 2016? Como objetivo general la investigación buscó: Demostrar la influencia de la aplicación del módulo Socializarte en las habilidades sociales avanzadas de estudiantes del segundo y tercer semestre de la Facultad de Ciencias de la Comunicación de la Universidad Nacional del Centro del Perú de Huancayo - 2016.

Como hipótesis general se planteó que: La aplicación del módulo Socializarte influye significativamente en las habilidades sociales avanzadas de estudiantes del segundo y tercer semestre de la Facultad de Ciencias de la Comunicación de la Universidad Nacional del Centro del Perú de Huancayo - 2016.
La investigación se fundamenta en la teoría de habilidades sociales de Goldstein, Sprafkin, Gershaw y Klein (1989), quienes clasificaron seis grupos de habilidades sociales: primeras habilidades sociales, habilidades sociales avanzadas, habilidades relacionadas con sentimientos, habilidades de planificación, habilidades para hacer frente al estrés y habilidades alternativas a la agresión. Goldstein et al. (1989), definieron las habilidades sociales avanzadas, como habilidades que desarrolla el individuo para relacionarse satisfactoriamente en entornos sociales, se desarrollan después de las primeras habilidades y ayudan a la persona a desenvolverse de manera práctica en la sociedad; están integradas por la habilidad para pedir ayuda, participar, dar instrucciones, seguir instrucciones, disculparse y convencer a los demás.

Kelly (2002), desde la teoría del aprendizaje social, definió a las habilidades sociales como conductas aprendidas. Así el desarrollo de la conducta de una persona, puede describirse, explicarse y predecirse con precisión por los principios de la teoría del Aprendizaje.

Las habilidades sociales se aprenden por el siguiente mecanismo: primero, la reflexión inicial con la presentación verbal de la habilidad que se quiere enseñar mediante dialogo en el contexto de sus vivencias, acciones o situaciones; segundo, la guía para practicar la habilidad con la instrucción verbal del proceso conductual que conforma la habilidad; tercero, el modelado de habilidades mediante ejemplos que en lo posible deben ser reales; cuarto, la práctica de la habilidad, primero en situaciones simuladas como role playing o dramatización y luego se traslada a situaciones naturales, espontaneas y cotidianas reforzando la habilidad; quinto, el feedback y reforzamiento donde los participantes aportan información de cómo la ha sido la práctica y se refuerza para la ejecución adecuada; sexto, la reflexión final a través del dialogo y debate y séptimo, la asignación de tareas para que el participante ejercite la habilidad en un contexto real y posterior revisión (Monjas, 2016).

El módulo educativo Socializarte, diseñado por el autor para la presente investigación, es una propuesta organizada de enseñanza aprendizaje, que tiene el propósito de potenciar, reforzar o desarrollar habilidades sociales avanzadas en estudiantes universitarios. La metodología didáctica se basa en el enfoque de aprendizaje estructurado (Goldstein et al, 1989), modelamiento y aprendizaje social (Bandura 1977). El módulo Socializarte fue organizado en torno a núcleos de contenidos, que se desarrollan en ocho sesiones. Un módulo educativo considera todos los elementos que se necesitan en el proceso de aprendizaje, que ha desarrollarse al ritmo del estudiante y sin la presencia continua del instructor Yukavetsky (2003). 
Verde (2015), al experimentar con una muestra de 21 estudiantes de primer grado de secundaria, concluyó que la aplicación el taller Aprendiendo a convivir, desarrolló significativamente el nivel de las habilidades sociales en los estudiantes del primer grado de educación secundaria de la I.E. Víctor Raúl Haya de la Torre de El Porvenir-Trujillo.

Lescano (2012), al experimentar con estudiantes de sexto grado de educación primaria, concluyó que la aplicación del taller educativo Renovando mis Valores, fortaleció significativamente las habilidades sociales de los estudiantes de sexto grado de la I. E № 00110 - San Francisco del Alto Mayo - Awajun.

Angulo (2012), al experimentar con estudiantes del sexto grado de educación primaria, concluyó que la aplicación del programa pedagógico Viviendo en armonía, desarrolló significativamente las habilidades sociales de los estudiantes del sexto grado de primaria de la I.E. Los Laureles del Distrito del Porvenir en Trujillo.

Moreno (2011), al experimentar con alumnos del primer grado de bachillerato en México, concluyó que el taller Entrenando mis Habilidades Sociales para la Vida, sí favorece la integración escolar.

Peres (2008), al experimentar con adolescentes institucionalizadas en España, concluyó que la aplicación de un programa de habilidades sociales, logró elevar y mejorar el afrontamiento con el entorno y las adolescentes obtuvieron herramientas que trasferirán a situaciones laborales y reinserción en el medio ambiente.

Choque (2006), al experimentar con estudiantes adolescentes de educación secundaria, concluyó que el programa educativo de habilidades para la vida es efectivo en un año escolar en el aprendizaje de las habilidades de comunicación y asertividad.

Llanos (2006), al experimentar con alumnos de primer, segundo y tercero de primaria en Bolivia, concluyó que la aplicación del programa Que Divertido es Aprender Habilidades Sociales, mejoró significativamente la competencia social.

El presente estudio al ser de tipo experimental, tiene productividad científica, y demuestra en forma fáctica los fundamentos de habilidades sociales; de tal forma que en la dinámica científica se constituye en un antecedente de estudio para posteriores investigaciones y referente cognitivo de las variables investigadas, también busca responder a la demanda en ámbito laboral de profesionales con adecuadas habilidades sociales como parte de las habilidades blandas y la necesidad de ciudadanos con capacidad para vincularse positivamente.
La investigación aporta con un modelo de la intervención psicopedagógica en la formación integral del estudiante en el nivel superior. Los resultados son referente fáctico que refuerzan las teorías psicopedagógicas que valoran la motivación y el aprendizaje significativo en el campo educativo. De similar manera el módulo educativo Socializarte y el cuestionario de habilidades sociales avanzadas diseñados y validados en la presente investigación podrán constituirse en herramientas para otros investigadores.

Como limitación del estudio, se consideró el factor subjetivo que se presenta al encuestarse al participante sobre su percepción respecto a sus habilidades sociales y factores del entorno de cada uno de los participantes que influiría en sus habilidades sociales. Para minimizar tales limitaciones, el estudio solo abordó las habilidades sociales avanzadas, se persuadió participantes para que sean lo más objetivo posible respecto a sus habilidades sociales avanzadas y se evaluó el pre test un mes después de haber acabado la experimentación, tiempo en el cual el grupo control haya podido interiorizar y practicar las habilidades sociales avanzadas.

\section{MATERIAL Y MÉTODOS}

La investigación por su finalidad es de tipo aplicada, según Sánchez y Reyes (2006), la investigación aplicada tiene como finalidad primordial la resolución de problemas prácticos inmediatos; en tal sentido el estudio sirvió para proponer la solución al problema de la debilidad de las habilidades sociales avanzadas en estudiantes la Facultad de Ciencias de la Comunicación de la Universidad Nacional del Centro del Perú y a partir de los resultados de la experimentación implementar la mejora educativa integral en la Facultad.

El diseño investigación cuasi experimental, analizó la influencia de la variable $X$ sobre la variable $Y$. Para, Hernández, Fernández y Baptista (2014), los diseños cuasi experimentales manipulan premeditadamente por lo menos una variable independiente para observar su efecto sobre una o más variables dependientes. En los diseños cuasi experimentales, los sujetos no se asignan al azar ni se emparejan, sino que ya están conformados antes del experimento.

El esquema es de la siguiente manera:

$$
\begin{array}{llll}
\text { GE: } & O_{1} & \times & O_{2} \\
\text { GC: } & O_{1} & & O_{2}
\end{array}
$$

Donde:

$\mathrm{GE}=30$ estudiantes del segundo semestre de la 
Tabla 1

Distribución de la muestra de estudio.

\begin{tabular}{cccccc}
\hline \multicolumn{1}{c}{ Grupo } & Estudiantes & Sem. II & Sem. III & Varones & Mujeres \\
\hline Experimental & 30 & 30 & 0 & 14 & 16 \\
control & 30 & 0 & 30 & 19 & 11 \\
\hline
\end{tabular}

Facultad de Ciencias de la Comunicación de la Universidad Nacional del Centro del Perú.

$\mathrm{GC}=30$ estudiantes del tercer semestre de la Facultad de Ciencias de la Comunicación de la Universidad Nacional del Centro del Perú.

$\mathrm{O}_{1}=$ Pre test al grupo experimental y al grupo control.

$X=$ A plicación del módulo Socializarte al grupo experimental.

$\mathrm{O}_{2}=$ Post test al grupo experimental y al grupo control.

La población estuvo constituida por 69 estudiantes del segundo y tercer semestre, matriculados en el periodo académico 2016-I en la Facultad de Ciencias de la Comunicación de la Universidad Nacional del Centro del Perú.

El muestreo no probabilístico, por conveniencia, se realizó según el siguiente criterio: los grupos de estudio fueron elegidos en base a las medias aritméticas del pre test (tabla 2), el grupo control con la media relativamente mayor $(74,87)$, los estudiantes del segundo semestre (30); el grupo experimental con la media relativamente menor $(74,5)$, los estudiantes del tercer semestre (30), tal como se detalla en la tabla 1. Como técnica de investigación se aplicó la encuesta, que por medio de preguntas dirigidas es empleada para investigar masivamente hechos o fenómenos y se utiliza principalmente en las ciencias sociales (Kerlinger, 1983).

Como instrumento, en el pre y post test se utilizó el cuestionario de habilidades sociales avanzadas diseñado para la investigación.

La validez del instrumento comprendió la validez de contenido y la validez de constructo; la primera, llamada también validez teórica, subjetiva o de escritorio, abarcó la validez genérica de criterios y la validez específica de ítems y, se evaluó mediante el juicio de cuatro expertos y con el coeficiente de concordancia $\checkmark$ de Aiken; logrando en el coeficiente de validez de Aiken $=1$ que implica validez perfecta; como resultado el cuestionario es válido. La segunda, llamada también validez práctica, objetiva o de campo, se realizó con los resultados de la aplicación del instrumento a una muestra piloto de 50 estudiantes del primero, quinto y sexto semestres semestres de la Facultad de Ciencias de la Comunicación la Universidad Nacional de Centro del Perú y, con el coeficiente de correlación item-test corregida $r$ de Pearson, como resultado como resultado, todos los coeficientes de correlación $r$ de Pearson, ítem-test, fueron mayores a 0,2 $(r \geq 0,2)$, por lo tanto, el cuestionario de habilidades sociales avanzadas es válido.

La confiabilidad del instrumento se evaluó en base a los resultados de su aplicación a una muestra piloto de 50 estudiantes del primero, quinto y sexto semestres de la Facultad de Ciencias de la Comunicación la Universidad Nacional de Centro del Perú y, con el coeficiente de consistencia interna alfa de Cronbach, el resultado obtenido fue de 0,904 , al ser mayor que 0,7 implica una confiabilidad excelente $(0,9$ a 1$)$, por lo que el instrumento es confiable.

\section{RESULTADOS}

La aplicación del módulo Socializarte en los estudiantes de la Facultad de Ciencias de la Comunicación de la Universidad Nacional del Centro del Perú en 2016, tuvo los siguientes resultados.

En la tabla 2 y figura 1 se aprecia que, en el pre test, ambos grupos de estudio tienen igual promedio en habilidades sociales avanzadas (experimental: 74,5; control: 74,87), mientras que, en el post test, el grupo

Tabla 2

Estadísticos de las habilidades sociales avanzadas por test y grupo $(n=60)$.

\begin{tabular}{clrr}
\hline Grupo de estudio & \multicolumn{1}{c}{ Estadístico } & Pre test & Post test \\
\hline Experimental & Media aritmética & $74,500 \%$ & 95,3 \\
$\mathrm{~N}=30$ & Desviación típica & $5,469 \%$ & 5,861 \\
& Coeficiente de variación & $7,300 \%$ & $6,2 \%$ \\
& & & \\
Control & Media aritmética & $74,870 \%$ & 75,87 \\
$\mathrm{~N}=30$ & Desviación típica & $4,232 \%$ & 4,904 \\
& Coeficiente de variación & $5,700 \%$ & $6,5 \%$ \\
\hline
\end{tabular}




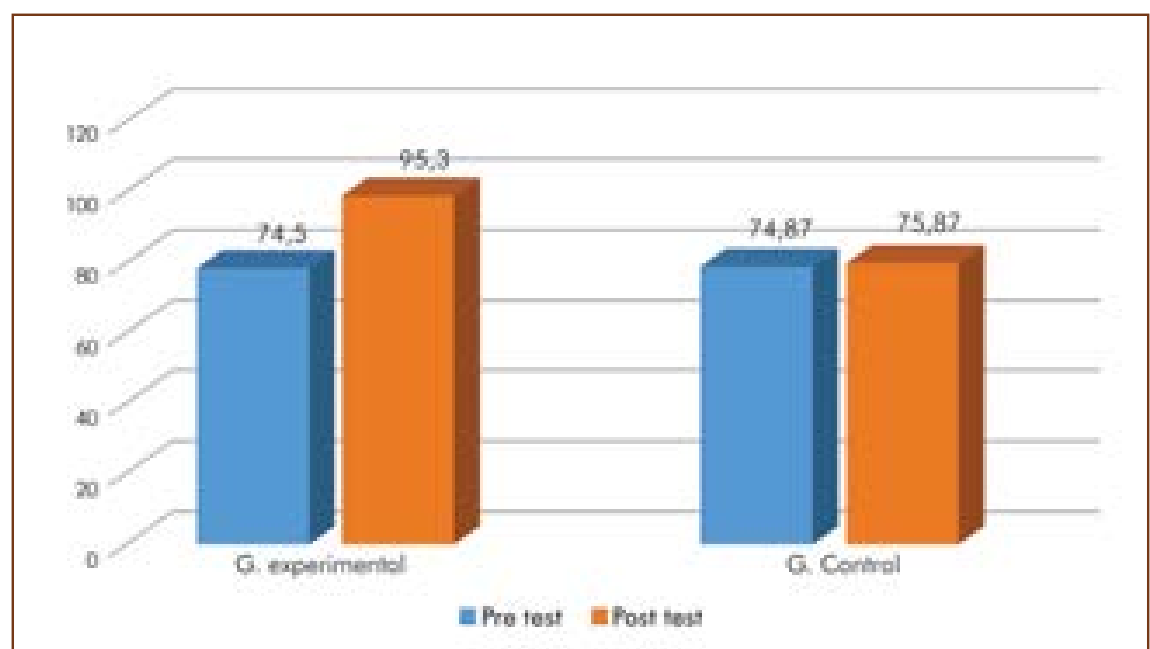

Figura 1. Comparación de las habilidades sociales avanzadas de los grupos experimental y control en el pre test y post test.

Tabla 3

Prueba de homogeneidad de varianzas y medias en el pre test.

\begin{tabular}{|c|c|c|c|c|c|}
\hline \multirow[t]{2}{*}{ Dimensión } & \multirow[t]{2}{*}{ Estadísticos ${ }^{1 /}$} & \multicolumn{2}{|c|}{$\begin{array}{c}\text { Prueba de homogeneidad } \\
\text { de varianzas }\end{array}$} & \multicolumn{2}{|c|}{$\begin{array}{l}\text { Prueba de homogeneidad } \\
\text { de medias }\end{array}$} \\
\hline & & Exper. & Control & Exper. & Control \\
\hline \multirow[t]{3}{*}{ Pedir ayuda } & Varianza / Media & 2,171 & 1,197 & 10,970 & 10,90 \\
\hline & Valor calculado & 1,810 & & 0,200 & \\
\hline & Valor P & 0,114 & & 0,843 & \\
\hline \multirow[t]{3}{*}{ Participar } & Varianza / Media & 2,800 & 1,689 & 12,400 & 12,37 \\
\hline & Valor calculado & 1,660 & & 0,090 & \\
\hline & Valor P & 0,179 & & 0,932 & \\
\hline Dar & Varianza / Media & 4,437 & 5,799 & 12,670 & 12,83 \\
\hline \multirow[t]{2}{*}{ instrucciones } & Valor calculado & 0,770 & & 0,290 & \\
\hline & Valor P & 0,475 & & 0,776 & \\
\hline Seguir & Varianza / Media & 3,421 & 2,740 & 13,600 & 13,53 \\
\hline \multirow[t]{2}{*}{ instrucciones } & Valor calculado & 1,250 & & 0,150 & \\
\hline & Valor P & 0,554 & & 0,884 & \\
\hline \multirow[t]{3}{*}{ Disculparse } & Varianza / Media & 2,162 & 2,144 & 11,900 & 11,83 \\
\hline & Valor calculado & 1,010 & & 0,180 & \\
\hline & Valor P & 0,982 & & 0,861 & \\
\hline Convencer a & Varianza / Media & 3,275 & 1,789 & 12,970 & 13,07 \\
\hline \multirow[t]{2}{*}{ los demás } & Valor calculado & 1,830 & & 0,240 & \\
\hline & Valor P & 0,109 & & 0,809 & \\
\hline Habilidades & Varianza / Media & 29,914 & 17,913 & 74,505 & 74,87 \\
\hline \multirow[t]{2}{*}{ sociales } & Valor calculado & 1,67 & & 0,290 & \\
\hline & Valor P & 0,173 & & 0,773 & \\
\hline
\end{tabular}

Fuente: Base de datos

1/Estadísticos de prueba: Homogeneidad de varianzas: F de Fisher; Homogeneidad de medias: t de Student

Exper.: Grupo experimental

Homogeneidad de varianzas: $G L=29$ y 29 ; Fteo(1) $=0,476$; Fteo(2) $=2,101$

Homogeneidad de medias: $G L=58 ; \operatorname{tteo}(1)=-2,002 ; \operatorname{tteo}(2)=2,002$ 
experimental $(95,3)$ logra mayor promedio que el grupo control $(75,87)$. Ambos grupos son homogéneos en ambos test.

Las medias de las habilidades sociales avanzadas en el pre test y post test de los estudiantes del grupo experimental son diferentes, favorables al post test.

Las medias de las habilidades sociales avanzadas
En la tabla 3 se aprecia que, en el pre test, los grupos de estudio (experimental y control) son homogéneos o iguales en varianzas y medias, tal como lo evidencian las pruebas $\mathrm{F}$ de Fisher para la homogeneidad de varianzas y $t$ de Student para la homogeneidad de medias de dos muestras independientes pequeñas.

En la tabla 4 se aprecia que, las medias de cada una de las habilidades sociales avanzadas de los

Tabla 4

Prueba t de Student para la diferencia de medias de dos muestras independientes para cada una de las habilidades sociales avanzadas en el post test.

\begin{tabular}{lccccccc}
\hline Habilidad & \multicolumn{2}{c}{$\begin{array}{c}\text { Grupo } \\
\text { experimental }\end{array}$} & \multicolumn{2}{c}{$\begin{array}{c}\text { Grupo } \\
\text { control }\end{array}$} & \multirow{2}{*}{$\begin{array}{c}\text { T } \\
\text { calcul. }\end{array}$} & $\begin{array}{c}\text { Valor } \\
\text { P }\end{array}$ \\
\cline { 2 - 5 } Media & DE & Media & DE & & \\
\hline Pedir ayuda & 15,90 & 1,49 & 11,03 & 1,13 & 14,24 & 0,000 \\
Participar & 16,27 & 1,76 & 12,47 & 1,59 & 8,77 & 0,000 \\
Dar instrucciones & 17,37 & 1,59 & 12,93 & 1,51 & 11,10 & 0,000 \\
Seguir instrucciones & 16,47 & 1,59 & 13,73 & 1,11 & 7,71 & 0,000 \\
Disculparse & 14,03 & 2,50 & 12,33 & 1,63 & 3,12 & 0,003 \\
Convencer a los demás & 15,27 & 1,60 & 13,37 & 1,00 & 5,53 & 0,000 \\
\hline
\end{tabular}

Fuente: Base de datos

Tabla 5

Prueba t de Student para la diferencia de medias de dos muestras relacionadas para cada una de las habilidades sociales avanzadas del grupo experimental.

\begin{tabular}{lccccccc}
\hline Habilidad & \multicolumn{2}{c}{$\begin{array}{c}\text { Pre } \\
\text { test }\end{array}$} & \multicolumn{2}{c}{$\begin{array}{c}\text { Post } \\
\text { test }\end{array}$} & & $\begin{array}{c}\text { T } \\
\text { calcul. }\end{array}$ & $\begin{array}{c}\text { Valor } \\
\text { P }\end{array}$ \\
\cline { 2 - 5 } & Media & DE & Media & DE & & \\
\hline Pedir ayuda & 10,97 & 1,47 & 15,90 & 1,49 & 14,01 & 0,000 \\
Participar & 12,40 & 1,67 & 16,27 & 1,76 & 8,47 & 0,000 \\
Dar instrucciones & 12,67 & 2,11 & 17,37 & 1,59 & 10,95 & 0,000 \\
Seguir instrucciones & 13,60 & 1,85 & 16,47 & 1,59 & 6,28 & 0,000 \\
Disculparse & 11,90 & 1,47 & 14,03 & 2,50 & 3,88 & 0,001 \\
Convencer a los demás & 12,97 & 1,81 & 15,27 & 1,60 & 4,55 & 0,000 \\
\hline
\end{tabular}

Fuente: Base de datos

en el pre test y post test de los estudiantes del grupo control son iguales.

En el post test, las medias de las habilidades sociales avanzadas de los estudiantes del grupo control y experimental son diferentes, favorables al grupo experimental. estudiantes del grupo control y experimental son diferentes, favorables al grupo experimental.

En la tabla 5 se aprecia que, las medias de cada una de las habilidades sociales avanzadas en el pre test y post test de los estudiantes del grupo experimental son diferentes, favorables al grupo experimental. 
Tabla 6

Prueba t de Student para la diferencia de medias de dos muestras relacionadas para cada una de las habilidades sociales avanzadas del grupo control.

\begin{tabular}{lccccccc}
\hline Habilidad & \multicolumn{2}{c}{$\begin{array}{c}\text { Pre } \\
\text { test }\end{array}$} & \multicolumn{2}{c}{$\begin{array}{c}\text { Post } \\
\text { test }\end{array}$} & & $\begin{array}{c}\text { T } \\
\text { calcul. }\end{array}$ & $\begin{array}{c}\text { Valor } \\
\text { P }\end{array}$ \\
\cline { 2 - 5 } & Media & DE & Media & DE & & \\
\hline Pedir ayuda & 10,90 & 1,09 & 11,03 & 1,13 & 0,49 & 0,631 \\
Participar & 12,37 & 1,30 & 12,47 & 1,59 & 0,24 & 0,811 \\
Dar instrucciones & 12,83 & 2,41 & 12,93 & 1,51 & 0,31 & 0,762 \\
Seguir instrucciones & 13,53 & 1,66 & 13,73 & 1,11 & 0,58 & 0,565 \\
Disculparse & 11,83 & 1,46 & 12,33 & 1,63 & 1,58 & 0,126 \\
Convencer a los demás & 13,07 & 1,34 & 13,37 & 1,00 & 1,09 & 0,286 \\
\hline
\end{tabular}

Fuente: Base de datos

En la tabla 6 se aprecia que, las medias de cada una de las habilidades sociales avanzadas en el pre test y post test de los estudiantes del grupo control son iguales.

\section{Contrastación de hipótesis}

El contraste de la hipótesis se realizó con la prueba † de Student para la homogeneidad de medias de dos muestras independientes y relacionadas, al $95 \%$ de confianza estadística. La prueba de homogeneidad de medias de dos muestras independientes, para comparar las medias de los grupos experimental y control, tanto en el pre test como en el post test y, la prueba de homogeneidad de dos muestras correlacionadas, para comparar las medias del pre test y post test, tanto en el grupo experimental como en el grupo control.

Se comprobó que, en el pre test, no existen diferencias significativas entre las medias de las habilidades sociales avanzadas de los grupos experimental y control ( $T=0,29$ y $p=0,773)$; se evidenció que, en el post test, existen diferencias significativas entre las medias de las habilidades sociales avanzadas de los grupos experimental y control, favorables al grupo experimental $(T=13,93$ y $p=0)$.

Por otra parte, se comprobó que existen diferencias significativas entre las medias de las habilidades sociales avanzadas del pre test y post test del grupo experimental, favorables al post test $(T=15,10 \mathrm{y}$ $\mathrm{p}=0$ ); se evidenció que, en el post test, no existen diferencias significativas entre las medias de las habilidades sociales avanzadas del grupo control ( $T=$ $1,68$ y $p=0,104)$.

Con los resultados se aceptó la hipótesis general de investigación, afirmando que la aplicación del módulo educativo Socializarte influye significativamente en las habilidades sociales avanzadas de estudiantes del segundo y tercer semestres de la Facultad de Ciencias de la Comunicación de la Universidad Nacional del Centro del Perú de Huancayo.

\section{DISCUSIÓN}

La investigación halló que, en el pre test, no existen diferencias significativas entre las medias de las habilidades sociales avanzadas de los grupos experimental y control. En el post test, sí existen diferencias significativas entre las medias de las habilidades sociales avanzadas de los grupos experimental y control, favorables al grupo experimental.

También se halló que existen diferencias significativas entre las medias de las habilidades sociales avanzadas del pre test y post test del grupo experimental, favorables al post test. Se comprobó que no existen diferencias significativas entre las medias de las habilidades sociales avanzadas del pre test y post test del grupo control.

A partir de los resultados, se acepta la hipótesis general de investigación, demostrándose que sí es posible lograr cambios significativos en las habilidades sociales avanzadas a través de la implementación adecuada de un módulo educativo, basado en la teoría de aprendizaje social de Bandura (1977), el enfoque de aprendizaje estructural como entrenamiento psicoeducacional para las habilidades sociales de Goldstein et al. (1989), y la técnica del modelado definida por Bandura y Walters (1990) para la modificación de conducta. También se demostró la posibilidad de medir las habilidades sociales 
avanzadas a través de un instrumento diseñado a partir del modelo teórico de Goldstein et al. (1989), que clasifica las habilidades sociales en seis grupos: habilidades sociales básicas o primeras habilidades sociales, habilidades sociales avanzadas, habilidades relacionadas con sentimientos, habilidades de planificación, habilidades para hacer frente al estrés y habilidades alternativas a la agresión.

Los resultados guardan relación con lo que obtuvieron las experimentaciones de Verde (2015), con alumnos del primer año de educación secundaria; Angulo (2012), con estudiantes del sexto grado de educación primaria; Lescano (2012), con estudiantes de sexto grado de educación primaria; Moreno (2011), con alumnos del primer grado de bachillerato en México; Peres (2008), con adolescentes institucionalizadas en España; Choque (2006), con estudiantes adolescentes de educación secundaria, y Llanos (2006) con alumnos de primer, segundo y tercero de primaria en Bolivia, la presente investigación también experimentó con éxito un programa de entrenamiento en habilidades sociales.

Sin embrago, también se presentan diferencias con las investigaciones experimentales referidas como antecedentes; la presente investigación abordó el nivel de educación superior en el ámbito universitario, enfocando los objetivos desde el punto de vista de la formación profesional, para lo cual se diseñó un módulo educativo de entrenamiento de habilidades sociales avanzadas y el instrumento de evaluación del mismo. Otra diferencia es que la presente investigación al considerar que las habilidades sociales integran un gran número de capacidades, solo se orientó al campo específico de las habilidades sociales avanzadas en base a la teoría de Goldstein ef al. (1989), quienes definieron las habilidades sociales avanzadas como aquellas se desarrollan después de las primeras habilidades, y son habilidades de las se requiere con mayor presencia en el ámbito laboral y desarrollo profesional. Se coincide con Angulo (2012), quien abordó específicamente las habilidades sociales básicas, con niños del nivel primario.

Las habilidades sociales son patrones conductuales aprendidos desde la infancia, por lo que son constantes y definen algunos rasgos de la personalidad. Con referencia a Monjas (2012) y Caballo (2007), quienes afirman que para mejorar las habilidades sociales no son eficaces la simple observación o instrucción informal, y que es imprescindible una instrucción directa y sistemática; se discrepa con los antecedentes que también basados en la teoría de Goldstein et al. (1989), abordaron como propósito de su investigación la mejora de los seis grupos de habilidades sociales en un corto periodo de tiempo.

Entrenar habilidades sociales no es tarea fácil, debe estar a cargo del profesional adecuado, que facilite a través de la técnica del modelamiento la adquisición de las nuevas conductas. Las corrientes psicológicas conductista, cognitivista y de aprendizaje social coinciden en que generar cambio de conducta es un proceso laborioso e implica teorías y técnicas adecuadas de la psicología que se deben abordar con conductas focalizadas en un plazo adecuado. El entrenamiento de las habilidades sociales, implica poner en práctica, en un plazo adecuado, estrategias de cambio de conducta, asegurando su sostenibilidad. Los seis tipos de habilidades sociales de Goldstein et al. (1989), definen aproximadamente 50 conductas, lo cual sería muy complicado de modificar a mediano plazo. Por tal motivo la presente investigación solo abordó las habilidades sociales avanzadas, que implica la modificación de seis conductas.

Los resultados de la investigación demuestran que en el grupo experimental, los índices de mejora de las habilidades para dar instrucciones y seguir, instrucciones, son mayores respecto a las habilidades para pedir disculpas, participar, pedir disculpas y convencer a los demás. En mérito a los resultados se aprueba la primera hipótesis de investigación, ello es lógico, porque pedir disculpas y convencer a los demás son habilidades que requieren mayor entrenamiento, refuerzo y tiempo respecto a las otras habilidades.

De similar manera, Moreno (2011), halló que su muestra tuvo mayor progreso en habilidades sociales como: pedir ayuda, manejar el estrés y manejo de emociones. Choque (2006), logró mejoras en las habilidades de comunicación y asertividad.

Los resultados de la investigación hallaron que en el post test del grupo experimental, hubo mejora significativa en las habilidades sociales avanzadas respecto al pre test del mismo grupo.

A diferencia de las investigaciones experimentales que sirvieron como antecedentes, en la presente investigación se evaluó el post test un mes después de haber culminado el entrenamiento; se consideró ese tiempo como el adecuado para que los participantes del grupo experimental pudieran adaptar su vida cotidiana las habilidades aprendidas y asegurar que el post test refleje el impacto de la experimentación y su sostenibilidad, también se evaluó al final de cada sesión de aprendizaje, para medir los logros por dimensión y tomar decisiones.

Respecto a la cantidad de muestra, la presente investigación trabajó con 30 estudiantes de nivel superior como grupo experimental, cantidad similar a la investigación de Moreno (2011), con 35 estudiantes de secundaria; Peres (2008), con 35 adolescentes y Angulo (2012), con 29 estudiantes de primaria, logrando todos, resultados exitosos. 
Partiendo de la teoría del efecto espectador de Darley y Latane (2007), que lo definieron como un fenómeno psicológico por el cual es menos probable que una persona intervenga en una situación cuando hay más personas que cuando se está solo. La cantidad de muestra la de la presente investigación fue suficiente, por tratarse de entrenamiento en habilidades sociales avanzadas a través de técnicas participativas; un número mayor de la muestra hubiera sido contraproducente para los fines de la investigación. Se diverge con las experimentaciones de Choque (2006) con 142 estudiantes de secundaria y Llanos (2006) con 250 estudiantes de primaria. Para que los aportes de la presente investigación se apliquen en una situación cotidiana de tutoría o psicopedagogía y tenga resultados positivos, se recomienda trabajar en grupos de 15 participantes.

Finalmente se considera que la presente investigación es un aporte que permitirá contribuir a futuras investigaciones y nuevos métodos de entrenamiento de las habilidades sociales avanzadas.

Las conclusiones son:

Se ha demostrado que la influencia de la aplicación del módulo educativo Socializarte en las habilidades sociales avanzadas de estudiantes del segundo y tercer semestre de la Facultad de Ciencias de la Comunicación de la Universidad Nacional del Centro del Perú de Huancayo - 2016, es significativa $\mathrm{T}=$ 15,10 y $p=0$.

Se ha determinado que las habilidades sociales avanzadas de mayor progreso en estudiantes del segundo y tercer semestre de la Facultad de Ciencias de la Comunicación de la Universidad Nacional del Centro del Perú son dar instrucciones 17,4 y seguir instrucciones 16,5.

Se ha determinado que la influencia de la aplicación del módulo educativo Socializarte en la habilidad para pedir ayuda, de estudiantes del segundo y tercer semestre de la Facultad de Ciencias de la Comunicación de la Universidad Nacional del Centro del Perú de Huancayo es significativa $T=14,01$ y $p$ $=0,000$.

Se ha determinado que la influencia de la aplicación del módulo educativo Socializarte en la habilidad para participar, de estudiantes del segundo y tercer semestre de la Facultad de Ciencias de la Comunicación de la Universidad Nacional del Centro del Perú de Huancayo es significativa $T=8,47$ y $p=0,000$.

Se ha determinado que la influencia de la aplicación del módulo educativo Socializarte en la habilidad para dar instrucciones, de estudiantes del segundo y tercer semestre de la Facultad de Ciencias de la
Comunicación de la Universidad Nacional del Centro del Perú de Huancayo es significativa $T=10,95$ y $\mathrm{p}$ $=0,000$.

Se ha determinado que la influencia de la aplicación del módulo educativo Socializarte en la habilidad para seguir instrucciones, de estudiantes del segundo y tercer semestre de la Facultad de Ciencias de la Comunicación de la Universidad Nacional del Centro del Perú de Huancayo es significativa $T=6,28$ y $\mathrm{p}=$ 0,000 .

Se ha determinado que la influencia de la aplicación del módulo educativo Socializarte en la habilidad para disculparse, de estudiantes del segundo y tercer semestre de la Facultad de Ciencias de la Comunicación de la Universidad Nacional del Centro del Perú de Huancayo es significativa $T=3,88$ y $\mathrm{p}=$ 0,001 .

Se ha determinado que la influencia de la aplicación del módulo educativo Socializarte en la habilidad para convencer a los demás, de estudiantes del segundo y tercer semestre de la Facultad de Ciencias de la Comunicación de la Universidad Nacional del Centro del Perú de Huancayo es significativa $T=4,55$ у $\mathrm{p}=$ 0,000 .

\section{REFERENCIAS BIBLIOGRÁFICAS}

Angulo, E. (2012). Programa pedagógico "viviendo en armonía" para desarrollar las habilidades sociales básicas delos estudiantes del sexto grado de educación primaria de la I.E. Los Laureles del Distrito el Porvenir. Tesis de maestría. Universidad Privada Antenor Orrego de Perú, Trujillo, Perú.

Ballester, R. y Gil Llario, M. (2002). Habilidades sociales. Teoría, investigación e intervención. Madrid: Síntesis.

Bandura, A. y Walters, R (1990). Aprendizaje social y desarrollo de la personalidad. Madrid: Alianza.

Bandura, A. (1977). Social learnig theory. New York: Gewneral Learnings Press.

Caballo, V. (2007). Manual de evaluación y entrenamiento de las Habilidades Sociales (Séptima ed.). Madrid: Siglo XXI.

Choque, R. (2006). Eficacia del programa educativo de habilidades para la vida en adolescentes de una institución educativa del distrito de Huancavelica, 2006. Tesis de maestría. Universidad Cayetano Heredia, Lima, Perú.

Darley M. y Latane B. (2007) Bystander intervention in emergencies: Diffusion of responsibility. New York: APA Journals

Goldstein A.; Sprafkin R.; Gershaw J. y Klein P. (1989) Habilidades sociales y autocontrol en 
la adolescencia. Un programa de enseñanza. Barcelona: Martínez Roca.

Hernández S. R.; Fernández C. C. y Baptista L. P. (2014) Metodología de la Investigación. México DF: Mc Graw Hill.

Kelly, J. (2002). Entrenamiento de las habilidades sociales (Séptima ed.). Bilbao: Descleé de Brouwer.

Kerlinger, F. y Lee, H. (2002) Investigación del comportamiento. (Cuarta ed.) México DF: Mc Graw Hill.

Lescano, J. (2012), Taller educativo "renovando mis valores" para fortalecer las habilidades sociales en los estudiantes de $6^{\circ}$ grado de educación primaria, área personal social de la I.E № 00110 - San Francisco del Alto Mayo - Awajun. Tesis de maestría. Universidad Nacional de San Martín, Tarapoto, Perú.

Llanos, C. (2006). Efectos de un programa de enseñanza en habilidades sociales. Tesis de doctorado. Universidad de Granada, Granada, España.

Monjas, I. (2016). Cómo Promover la Convivencia: Programa de Asertividad y Hablidades Sociales (PAHS). (Cuarta ed.). Madrid: CEPE.

Moreno, M. (2011). Desarrollo de habilidades sociales como estrategia de integración al bachillerato. Tesis de maestría. Universidad Autónoma de Yucatán, Yucatán, México.

Peres, M. (2008). Habilidades sociales es adolescentes institucionalizadas para el afrontamiento de su entorno inmediato. Tesis de doctorado. Universidad Granada, Granada, España.

Sánchez C. H y Reyes M. R. (2006) Metodología y Diseños en la Investigación Científica. Lima: Universidad Ricardo Palma.

Segura, M. (2007). Enseñar a convivir no es tan difícil. Para quienes no saben qué hacer con sus hijos o con sus alumnos. Bilbao: Desclée de Brouwer

Verde, R. (2015), Taller aprendiendo a convivir para el desarrollo de habilidades sociales en los alumnos del primer año de educación secundaria de la I.E. Víctor Raúl Haya de la Torre El Porvenir - Trujillo, 2014. Tesis de maestría, Universidad Privada Antenor Orrego, Trujillo, Perú.

Yukavetsky, G. (2003). La elaboración de un módulo instruccional. Humacao: Universidad de Puerto Rico. 